\title{
Rentabilidad de la pulsioximetría para el control de la oxigenoterapia domiciliaria
}

\author{
M. N. FEU COLLADO, B. JURADO GÁMEZ, J. RUBIO SÁNCHEZ, N. PASCUAL \\ MARTÍNEZ, A. A. ESCRIBANO DUEÑAS, F. GARCÍA GIL, L. MUÑOZ CABRERA
}

Servicio de Neumología. Hospital Universitario Reina Sofía. Córdoba

\author{
VALUE OF PULSE OXYMETRY FOR THE FOLLOW-UP CONTROL \\ OF DOMICILIARY OXYGEN THERAPY
}

\begin{abstract}
RESUMEN
Objetivo: Evaluar la oxigenoterapia continua domiciliaria (OCD) en nuestro medio y la rentabilidad de la pulsioximetría en su control.

Pacientes y método: Se estudian 140 pacientes. Los datos sobre indicación y grado de cumplimentación de la OCD quedan registrados mediante entrevista clínica, además se les realiza espirometría, hematimetría, gasometría arterial y pulsioximetría.

Resultados: Fueron excluidos 12 pacientes $(8,6 \%)$ que cumplían criterios de oxigenoterapia provisional y $22(15,7 \%)$ que presentaban agudización. De los 106 individuos restantes, 67 eran hombres $(63 \%)$ y 39 mujeres $(37 \%)$, edad media de $73 \pm 12$ años. El $54 \%$ de los enfermos (57/106) mantenían la oxigenoterapia al menos 15 horas/ día, sólo 36 pacientes $(34 \%)$ cumplían criterios de indicación de OCD y únicamente $24(23 \%)$ presentaban criterios de indicación y adecuada administración de la OCD. La saturación de oxígeno determinada por pulsioximetría $\left(\mathrm{SpO}_{2}\right)$ mostró una buena correlación con la presión arterial de oxígeno $\left(\mathrm{PaO}_{2}\right)$ obtenida por gasometría arterial $(\mathrm{r}=0,857$ y $\mathrm{K}=0,937)$.

Conclusiones: En nuestro medio, el control y cumplimiento de la OCD no es óptimo. La $\mathrm{SpO}_{2}$ muestra una buena correlación con la $\mathrm{PaO}_{2}$ y representa una buena alternativa para el control de la OCD.
\end{abstract}

PALABRAS CLAVE: Enfermedad pulmonar obstructiva crónica (EPOC). Oxigenoterapia crónica domiciliaria (OCD). Pulsioximetría.
ABSTRACT

Objective: To evaluate long-term domiciliary oxygen therapy (LTOT) in our setting and the utility of pulse oxymetry for follow-up control.

Patients and method: 140 patients were studied. The data on the indication for LTOT and the degree of therapeutic compliance were obtained by means of a clinical interview; spirometry, full blood count, arterial blood gas measurement, and pulse oxymetry were also carried out.

Results: $12(8.6 \%)$ patients who fulfilled criteria for provisional oxygen therapy were excluded and $22(15.7 \%)$ due to recent exacerbations. Of the remaining 106 individuals, 67 (63\%) were men and 39 (37\%) women, and the mean age was $73 \pm 12$ years. $54 \%$ (57/106) of the patients used the oxygen therapy for at least 15 hours/day. Only 36 (34\%) patients fulfilled the indication criteria for LTOT and only $24(23 \%)$ both fulfilled the indication criteria and demonstrated appropriate administration of the LTOT. The oxygen saturation measured by pulse oxymetry $\left(\mathrm{SpO}_{2}\right)$ showed a good correlation with the partial pressure of oxygen $\left(\mathrm{PaO}_{2}\right)$ obtained by arterial blood gas measurement ( $r=0.857$ and $K=0.937$ ).

Conclusions: In our setting, the follow-up control of LTOT and therapeutic compliance were not optimal. The $\mathrm{SpO}_{2}$ shows a good correlation with the $\mathrm{PaO}_{2}$ and represents a good alternative for the follow-up control of LTOT.

KEY WORDS: Chronic obstructive pulmonary disease (COPD). Chronic domiciliary oxygen therapy (CDO). Pulse oxymetry.

Feu Collado MN, Jurado Gámez B, Rubio Sánchez J, Pascual Martínez N, Escribano Dueñas AA, García Gil F, Muñoz Cabrera L. Rentabilidad de la pulsioximetría para el control de la oxigenoterapia domiciliaria. An Med Interna (Madrid) 2004; 21: 56-61.

\section{INTRODUCCIÓN}

Mediante el estudio epidemiológico IBERPOC, la prevalencia estimada en España de enfermedad pulmonar obstructiva crónica (EPOC) es del 9\% en el grupo etario de 40-69 años (1), esto representa en nuestro país aproximadamente 1.200.000 personas con EPOC que generan un gasto sanitario muy elevado (2). Actualmente en España supone la quinta causa de muerte en hombres y la séptima en mujeres, siendo por tanto un grave problema de salud pública.
La principal indicación de la oxigenoterapia es corregir la hipoxia tisular al aumentar la fracción inspirada de oxígeno. La oxigenoterapia crónica domiciliaria (OCD) es un tratamiento consolidado en las fases más avanzadas de la EPOC y esta plenamente establecido que administrada durante más de 15 horas al día mejora la supervivencia de los pacientes con EPOC en situación de insuficiencia respiratoria crónica $(3,4)$.

Se han publicado diversas normativas con la finalidad de establecer los criterios sobre la indicación y control posterior de la oxigenoterapia $(5,6)$. Sin embargo en los trabajos des-

Trabajo aceptado: 4 de septiembre de 2003

Correspondencia: M. N. Feu Collado. Servicio de Neumología. Hospital Universitario Reina Sofía. Avda. Menéndez Pidal, s /n. 14004 Córdoba. 
criptivos sobre OCD, es frecuente concluir que la prescripción de OCD y el cumplimiento por parte del paciente son insuficientes o inadecuados (7-10).

Por lo tanto en un programa de OCD, es conveniente la realización de revisiones periódicas con objeto de mejorar el cumplimiento de la terapia, reevaluar la indicación y controlar la corrección de la hipoxemia mediante el flujo de oxígeno prescrito. En este contexto la pulsioximetría representa un método no invasivo y rápido que permite conocer la saturación de oxígeno $\left(\mathrm{SpO}_{2}\right)$ de la hemoglobina, de esta forma parece razonable la utilización de la misma en el control de la OCD.

Nuestro objetivo fue conocer el uso de la OCD en nuestro medio, así como, valorar en las revisiones de los pacientes la utilidad de la pulsioximetría para determinar la continuidad o retirada de dicha terapéutica.

\section{MATERIAL Y MÉTODOS}

La revisión de la OCD se realiza en nuestro servicio en una consulta monográfica. Nuestra área sanitaria cuenta con una población de unos 500.000 habitantes y presenta como principales características sociodemográficas una población envejecida con una economía basada en la actividad agrícola y de servicios.

Se trata de un estudio observacional, transversal y abierto, durante el periodo comprendido entre el 15 de agosto 2001 al 15 de enero del año 2002, en el que se incluyeron a todos los pacientes que acudieron de forma programada a nuestra consulta.

Los criterios de inclusión fueron: edad mayor de 18 años, tratamiento con OCD y encontrarse en estabilidad clínica en los últimos tres meses.

Fueron excluidos del estudio a los enfermos que habían presentado exacerbación de su proceso respiratorio en los dos meses anteriores, los que referían una duración de la oxigenoterapia inferior a tres meses (indicación provisional) y aquellos con oxígeno líquido como forma de administración de la OCD, ya que estos son revisados específicamente en otra consulta.

Se consideró que la indicación de oxigenoterapia era correcta, cuando a pesar de un tratamiento broncodilatador adecuado se observaba hipoxemia crónica, esto es una presión arterial de oxígeno $\left(\mathrm{PaO}_{2}\right)$ basal menor o igual a $55 \mathrm{mmHg}$, o bien una $\mathrm{PaO}_{2}$ entre 55 y $60 \mathrm{mmHg}$ en presencia de cor pulmonale, insuficiencia cardiaca congestiva o poliglobulia (hematocrito $>55 \%$ ).

El tratamiento en la obstrucción crónica al flujo aéreo se juzgó adecuado si incluía la prescripción de al menos broncodilatadores inhalados (bromuro de ipratropio y /o beta2-agonistas) pautados y administrados correctamente, además de teofilinas y corticoides inhalados u orales según criterio del facultativo responsable. En las restantes patologías asumimos que la medicación estaba correctamente prescrita. En los enfermos con tratamiento broncodilatador y/o antinflamatorio inhalado se comprobó su correcta administración.

El cumplimiento de la OCD fue aceptado como bueno si el paciente se conectaba al $\mathrm{O}_{2}$ durante 15 o más horas al día y se consideró una correcta utilización de la OCD cuando se comprobaba que la indicación y cumplimentación era adecuada.

Se recogieron datos sobre la prescripción inicial (diagnós- tico de la enfermedad, servicio que indicó la OCD, gasometría arterial previa, número de horas recomendadas, fuente de administración, dispositivo utilizado, estabilidad clínica en el momento de la indicación), seguimiento (número de revisiones, tratamiento y correcta utilización de fármacos, existencia de poliglobulia y/o cor pulmonale, tabaquismo) y grado de cumplimiento de la OCD.

A todos los pacientes se les efectuó en el mismo día y por este orden: una hematimetría, determinando recuento celular, fórmula leucocitaria y hematocrito, pulsioximetría en reposo durante al menos 15 minutos (se aceptó aquel valor de $\mathrm{SpO}_{2}$ que se mantuvo estable durante 120 segundos), gasometría arterial en reposo y respirando aire ambiente, midiendo $\mathrm{pH}$, presión arterial de oxígeno $\left(\mathrm{PaO}_{2}\right)$, presión arterial de anhídrido carbónico, bicarbonato y saturación arterial de oxígeno $\left(\mathrm{SaO}_{2}\right)$. Por último se realizó una espirometría forzada para determinar la capacidad vital forzada (CVF) y volumen espiratorio forzado en el primer segundo (FEV1), siguiendo siempre las recomendaciones actualmente aceptadas $(11,12)$.

Para el registro de la pulsioximetría se utilizó un aparato Pulxox-7 Minolta, Co, Ltd Japan y en la espirometría forzada un espirómetro Vitalograf $2120^{\circledR}$.

Posteriormente dos neumólogos experimentados, desconociendo los resultados de la gasometría, evaluaron la situación clínica y los valores de la pulsioximetría y, a través de esta información, emitieron un juicio sobre la continuidad o interrupción de la OCD. Finalmente esta decisión era nuevamente evaluada, y se hacía definitiva, teniendo en cuenta los parámetros de la gasometría.

Los resultados obtenidos fueron registrados en una base de datos creada en el programa informático SPSS V 11.1 con el que se realizó el análisis estadístico. Las variables cualitativas fueron expresadas en valor absoluto y porcentaje, las cuantitativas como media y desviación estándar. Para comparar las frecuencias de las variables cualitativas se realizaron pruebas Chi cuadrado $\left(\chi^{2}\right)$ para tabla de contingencia $2 \times 2$ y prueba exacta de Fisher cuando alguna frecuencia esperada era inferior a 5. Se calcularon la sensibilidad, especificidad y valores predictivos positivo y negativo de la pulsioximetría respecto a la gasometría arterial, considerando a esta como el gold estándar. Se midió el índice de concordancia Kappa de Cohen entre la $\mathrm{PaO}_{2}$ obtenida por gasometría arterial y la $\mathrm{SpO}_{2}$ por pulsioximetría. También se obtuvo el coeficiente de correlación lineal de Pearson entre las variables $\mathrm{PaO}_{2}$ y $\mathrm{SpO}_{2}$. Todas las correlaciones realizadas fueron bilaterales y se consideró valores estadísticamente significativos cuando la $\mathrm{p}<0,05$.

\section{RESULTADOS}

De los 140 enfermos con OCD revisados se excluyeron a 34. Doce de ellos por presentar criterios de oxigenoterapia provisional y 22 por agudización respiratoria reciente. Por tanto, la muestra la componen 106 pacientes, 67 hombres $(63 \%)$ y 39 mujeres $(37 \%)$ con una edad media de $73 \pm 12$ años (23-93).

Los diagnósticos más frecuentes fueron: enfermedad pulmonar obstructiva crónica (EPOC) con un 67\% de los casos, enfermedad restrictiva (15\%) e insuficiencia cardiaca (13\%). En la tabla I se exponen los datos sociodemográficos y analíticos más relevantes según los distintos procesos diagnósticos. En la figura 1 se muestra la distribución de frecuencias según el 
TABLA I

DATOS SOCIODEMOGRÁFICOS Y ANALÍTICOS DE LOS PACIENTES ESTUDIADOS $(n=106)$ SEGÚN EL DIAGNÓSTICO (1)

\begin{tabular}{|c|c|c|c|c|}
\hline & $\begin{array}{l}\text { EPOC } \\
n=71\end{array}$ & $\begin{array}{c}\text { Enfermedad } \\
\text { restrictiva } \\
n=16\end{array}$ & $\begin{array}{c}\text { Insuficiencia } \\
\text { cardiaca } \\
n=14\end{array}$ & $\begin{array}{c}\text { Otras (2) } \\
n=5\end{array}$ \\
\hline $\operatorname{Sexo}(H / M)$ & $\begin{array}{l}57(80 \%) \\
14(20 \%)\end{array}$ & $\begin{array}{l}6(37 \%) / \\
10(63 \%)\end{array}$ & $\begin{array}{l}2(14 \%) / \\
12(86 \%)\end{array}$ & $\begin{array}{c}2(40 \%) / \\
3(60 \%)\end{array}$ \\
\hline Edad (años)* & $74 \pm 9$ & $65 \pm 18$ & $75 \pm 14$ & $64 \pm 8,5$ \\
\hline Tabaquismo & $13(18 \%)$ & 0 & $1(7 \%)$ & $1(20 \%)$ \\
\hline Controles periódicos & 42 & 9 & 6 & 5 \\
\hline Adecuada indicación OCD (3) & $22(31 \%)$ & $6(37 \%)$ & $6(43 \%)$ & $4(80 \%)$ \\
\hline Cumplimiento correcto OCD & $37(52 \%)$ & $8(50 \%)$ & $9(64 \%)$ & $3(60 \%)$ \\
\hline Tiempo OCD (meses)* & $44,2 \pm 44,6$ & $34,5 \pm 37,3$ & $23,4 \pm 14,7$ & $27 \pm 8,4$ \\
\hline $\operatorname{FVC~(4)~}(L) *$ & $1,92 \pm 0,70$ & $1,42 \pm 0,76$ & $1,33 \pm 0,74$ & $1,71 \pm 0,88$ \\
\hline FEV1 (5) (L)* & $0,90 \pm 0,38$ & $0,87 \pm 0,49$ & $0,84 \pm 0,35$ & $1,13 \pm 0,61$ \\
\hline $\mathrm{PaO}_{2}(6)(\mathrm{mmHg})^{*}$ & $65 \pm 11,7$ & $63,8 \pm 10,8$ & $67 \pm 14,4$ & $71,3 \pm 5,7$ \\
\hline $\mathrm{PCO}_{2}(7)(\mathrm{mmHg}) *$ & $41 \pm 7,2$ & $44,2 \pm 7,4$ & $41 \pm 4,9$ & $41 \pm 4,3$ \\
\hline
\end{tabular}

(1) Salvo indicación expresa, los resultados que se muestran son frecuencias absolutas y porcentajes. $\left(^{*}\right)$ indican $m \pm D S$.

(2) Otras patologías = 3 pacientes con alteraciones de la caja torácica y 2 con síndrome de Dawn e hipertensión pulmonar en situación de Eisenmenger.

(3) $\mathrm{OCD}=$ Oxigenoterapia continua domiciliaria

(4) FVC = Capacidad vital forzada.

(5) FEV1 = Volumen espirado forzado en el primer segundo.

(6) $\mathrm{PaO}_{2}=$ Presión arterial de oxígeno.

(7) $\mathrm{PaCO}_{2}=$ Presión arterial de anhídrido carbónico.

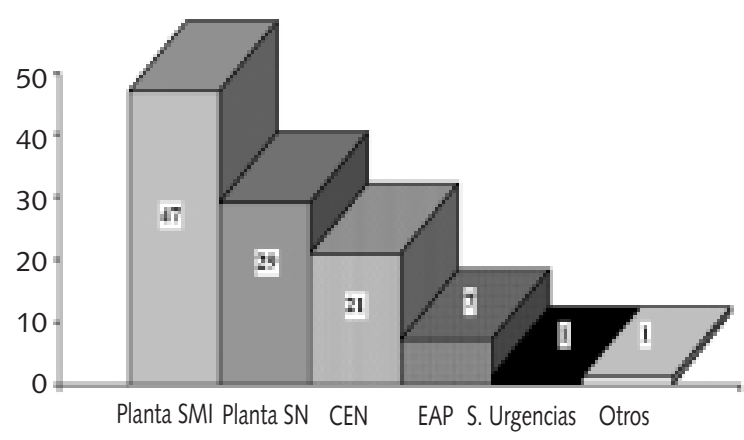

Fig. 1. Origen de la primera prescripción de OCD.

SMI: Servicio de Medicina Interna. SN: Servicio Neumología. CEN: Consulta Externa Neumología. EAP: Equipo de Atención Primaria.

nivel asistencial que realizó la primera prescripción de OCD.

Al 94\% (100/106) de los enfermos se les practicó una gasometría previa a la indicación de la OCD. La fuente de suministro más utilizada fue el cilindro de oxígeno a presión (89\%) y las gafas nasales el dispositivo de administración en el $100 \%$ de los casos, aunque 2 pacientes utilizaban además mascarilla tipo Ventimask a una $\mathrm{FiO}_{2}$ de 0,24 . El oxígeno se pautó a un flujo de $1 \mathrm{l} / \mathrm{m}$ en 19 enfermos (18\%), a 1,5 1/m en $68(64 \%)$ y a $21 / \mathrm{m}$ en $19(18 \%)$.
En 27 pacientes $(25,5 \%)$ la indicación de OCD se hizo en estabilidad clínica, mientras que en los 79 restantes $(74,5 \%)$ lo fue tras una agudización, posteriormente a sólo el $43 \%$ de estos (34/79) se les revisó nuevamente la indicación de la OCD.

El tiempo medio de administración de oxígeno fue de 13,4 \pm 5 horas (1-24), siendo de al menos 15 horas al día en el $54 \%$ de los pacientes (57/106).

El control de la enfermedad de base se llevó a cabo mediante los siguientes fármacos: beta-2 agonistas (73/106, $69 \%$ ), bromuro de ipratropio $(56 / 106,53 \%)$, y la asociación de ambos fármacos en 47 casos (44\%). A 56 enfermos (53\%) se les prescribió teofilinas de liberación retardada y a 53 $(50 \%)$ un corticoide inhalado. En 79 enfermos $(74,5 \%)$ el tratamiento estaba correctamente prescrito y pautado, sin embargo, la técnica de inhalación sólo era correcta en el $63 \%$ de ellos (50/79).

Respecto al consumo de tabaco 15 pacientes (14\%) reconocieron ser fumadores activos.

El 58,5\% (62/106) de los individuos seguían revisiones periódicas con control de gasometría arterial. De estos, 47 mantenían un tratamiento farmacológico adecuado (76\%) y 37 realizaban un cumplimiento correcto de la OCD (60\%), aunque estos resultados no alcanzaron un grado de significación estadística frente al grupo que no se revisaba periódicamente.

En el momento del estudio, el $36 \%$ de los pacientes (38/106) cumplían los criterios de OCD establecidos previamente y sólo el 23\% (24/106) realizaban además una adecuada cumplimentación de la OCD. 
Respecto a los valores de la gasometría y pulsioximetría, el análisis se realizó sobre 105 individuos ya que uno no aceptó la realización de la prueba, de ellos 17 presentaban una $\mathrm{PaO}_{2} \leq 55 \mathrm{mmHg}, 21$ una $\mathrm{PaO}_{2}$ entre 56-59 mmHg, y 67 una $\mathrm{PaO}_{2} \geq 60 \mathrm{mmHg}$. Las cifras de la $\mathrm{SpO}_{2}$ medida por pulsioximetría fueron en 10 pacientes menor o igual a $88 \%$, en 25 estuvo entre $89-92 \%$ y en 70 casos fue mayor a $92 \%$.

Para conocer la validez de la pulsioximetría en términos de sensibilidad y especificidad frente a la $\mathrm{PaO} 2$ medida por gasometría arterial, se aplicó como valor límite para el mantenimiento de la oxigenoterapia una $\mathrm{PaO}_{2} \leq 60 \mathrm{mmHg}$ y una $\mathrm{SpO}_{2}$ medida por pulsioximetría $\leq 92 \%$. El estudio muestra que sólo un paciente con una $\mathrm{PaO}_{2} \leq 60 \mathrm{mmHg}$. presentó una $\mathrm{SpO}_{2}$ superior al $92 \%$.

De esta forma la sensibilidad obtenida fue del $97 \%$ y la especificidad del $100 \%$, siendo el valor predictivo positivo del $100 \%$ y el valor predictivo negativo del $98 \%$.

Desde el punto de vista cualitativo, para conocer la concordancia o medida de acuerdo entre ambas pruebas se calculó el índice Kappa de Cohen y obtuvimos una $\mathrm{K}=0,937$ $(\mathrm{p}<0,001)$. En la valoración cuantitativa se observó una fuerte correlación entre ambas variables $(r=0,857$; $\mathrm{p}<0,001)$ (Fig. 2).

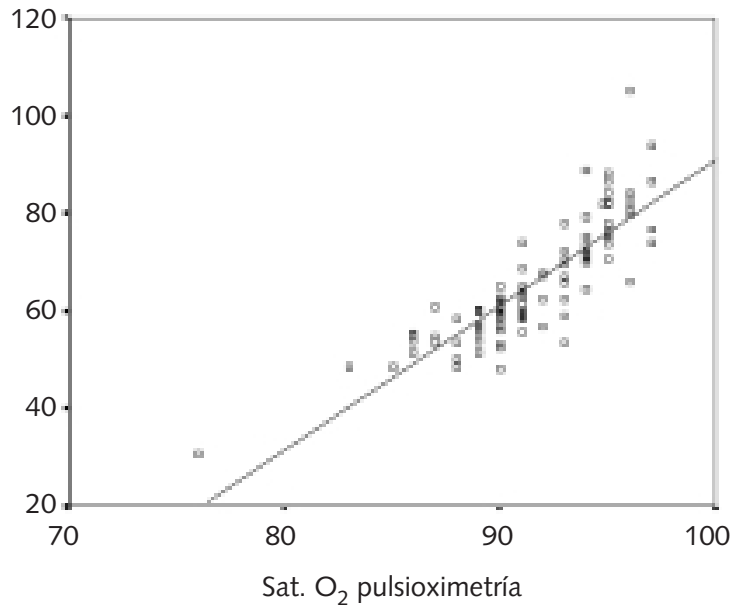

Fig. 2. Correlación observada entre la $\mathrm{PaO}_{2}$ aportada por la gasometría y la $\mathrm{SpO}_{2}$ determinada mediante pulsioximetría.

\section{DISCUSIÓN}

En nuestro estudio la OCD es un tratamiento con un inaceptable control e inadecuada cumplimentación. En este sentido los resultados de la pulsioximetría como técnica no invasiva y disponible en cualquier escalón asistencial han demostrado una excelente correlación con los valores gasométricos $y$, por tanto, constituye una herramienta que puede ayudar a optimizar el control de los pacientes en programa de OCD.

Aunque se sospecha que los beneficios de la oxigenoterapia puedan ser obtenidos en otras enfermedades del pulmón en situación de insuficiencia respiratoria crónica $(13,14)$, ha sido en la EPOC donde además de reducir su mortalidad, mejora la tolerancia al ejercicio (15), la poliglobulia (3), y la hipertensión pulmonar $(15,16)$.

A los beneficios de la OCD se contrapone un elevado coste económico y cierta dificultad de aceptación en algunos pacientes por el cambio de vida que les supone y que puede condicionar una mala cumplimentación, por lo tanto es obligado realizar una cuidadosa indicación y empleo de dicha terapéutica.

En nuestro trabajo la EPOC fue la patología más frecuente, $67 \%$ de los casos, porcentaje similar al de otros estudios (8-10,17-19). Llama la atención un alto número (13\%) de pacientes diagnosticados de insuficiencia cardiaca, si bien en su mayoría presentaban además un trastorno de la ventilación obstructivo como se deduce de los valores de la espirometría expresados en la tabla I.

Los broncodilatadores más utilizados fueron los beta-agonistas, seguido por el bromuro de ipratropio, solos o en combinación. Estos datos son los usuales en el control de la EPOC en la Comunidad de Andalucía (20) y que están en concordancia con las últimas recomendaciones $(5,6,21)$. Hay que destacar, sin embargo que sólo el 53\% de nuestros sujetos se encontraban con un tratamiento adecuado y con una técnica inhaladora correcta. Por lo tanto, casi la mitad de los pacientes, un óptimo control terapéutico de la enfermedad de base pudiera mejorar el intercambio gaseoso y replantear la necesidad de la OCD.

La técnica de inhalación incorrecta es otro de los problemas frecuentemente observado en la EPOC, la aplicación de los broncodilatadores ha sido estudiada en otros trabajos donde alcanzan cifras similares $(10,22)$. En la EPOC en estadios avanzados se ha demostrado que, a pesar de la gravedad clínica, existe frecuentemente una mala cumplimentación terapéutica (23). En este sentido probablemente haya que hacer mayor hincapié, teniendo en cuenta que los broncodilatadores no son efectivos si no se logra el cumplimiento por parte del paciente y una adecuada técnica inhalatoria (24).

La administración de oxígeno mediante concentrador esta bastante extendida en Europa (25), sin embargo, en nuestro medio representa un bajo porcentaje de utilización, dato que hay que valorar, ya que estas fuentes semiportátiles pueden influir positivamente en el cumplimiento de la OCD.

Es importante la detección de cambios clínicos o funcionales que puedan variar la indicación de la OCD, para ello es recomendable realizar controles periódicos en los pacientes con OCD, probablemente cada 6 meses (26). Paradójicamente observamos que los enfermos con dichas revisiones no son mejores cumplidores de la OCD, posiblemente debido a que en el seguimiento ni el médico ni el personal de enfermería enfatizan suficientemente la importancia de dicha terapéutica.

La primera indicación de la OCD debería basarse en los resultados de la gasometría arterial (12,27-29), en nuestra población en un $94 \%$ de los casos admitieron que fue así. Este alto porcentaje refleja que en su mayoría la indicación se efectuó en el ámbito hospitalario (Fig. 1), aspecto que ha sido corroborado en otros estudios $(8,9)$, por lo tanto, muchos de los pacientes presentaban inicialmente criterios gasométricos de OCD, sin embargo en el momento de realizar el estudio, 68 enfermos $(64 \%)$ no mantenían dicha indicación, este alto porcentaje es similar al encontrado en otros trabajos $(8,30,31)$. Los beneficios de la OCD sólo pueden esperarse si están pre- 
sentes hipoxemia crónica, corrección de la misma con el flujo prescrito y un adecuado cumplimiento $(5,7,27)$. En nuestro estudio sólo 24 pacientes (23\%) cumplían dichas premisas y, aunque estos bajos porcentajes son los observados por otros autores $(7-10,31,32)$, deben hacernos reflexionar sobre el inadecuado control que se mantiene de la EPOC en fase avanzada.

La $\mathrm{SpO}_{2}$ determinada por pulsioximetría se correlaciona bien con los valores medidos en sangre arterial (28), pero no puede considerarse a esta técnica como sustituta de la gasometría arterial en la indicación inicial de la OCD, momento en el que es importante la evaluación de la hipercapnia; sin embargo, es un buen método para el control de dicha oxigenoterapia y puede además utilizarse para ajustar el flujo de oxígeno (27-29). Mediante pulsioximetría, Morrison et al (33) valoran el adecuado flujo de oxígeno en pacientes con EPOC y detectan una inadecuada oxigenación en muchos de ellos.

En nuestro trabajo la $\mathrm{SpO}_{2}$ presenta una excelente correlación con los valores de $\mathrm{PaO}_{2}$ obtenidos por gasometría arterial. Empleando la pulsioximetría para indicar el mantenimiento o retirada de oxígeno y utilizando el punto de corte de la $\mathrm{SpO}_{2}$ en el 92\% (34-37), encontramos sólo un paciente que presentando una $\mathrm{SpO}_{2}$ del $93 \%$ tenía una $\mathrm{PaO}_{2}$ de $59 \mathrm{mmHg}$, no obstante, este mostraba signos clínicos de cor pulmonale crónico y, por tanto, teniendo en cuenta los criterios de indicación de OCD no era candidato a la retirada de la oxigenoterapia.

En cualquier caso, antes de tomar alguna decisión, siem- pre hay que valorar aquellas circunstancias que pueden producir error en la medida de la $\mathrm{SpO}_{2}$, como son la posición incorrecta de la sonda en el dedo, onda de pulso débil (bajo gasto, vasoconstricción cutánea), fuerte pigmentación cutánea, uñas pintadas etc.

Como recomiendan otros grupos (38), en aquellos pacientes con cifras bordeline de $\mathrm{SpO}_{2}$ igual a $92 \%$, además de valorar el contexto clínico, es aconsejable realizar una gasometría arterial antes de decidir el mantenimiento o retirada de la oxigenoterapia, mientras un valor en la $\mathrm{SpO}_{2} \geq 93 \%$ debe hacer replantear seriamente la indicación de la misma.

En los enfermos con EPOC grave hay que hacer hincapié en el tratamiento de la obstrucción bronquial de la enfermedad de base, así como, en el abandono del consumo de tabaco previo a la prescripción de OCD, única actitud terapéutica efectiva para frenar la caída progresiva del FEV1 en la EPOC $(5,6,21,39)$.

Cuando la insuficiencia respiratoria crónica está presente, su corrección mediante el aporte de $\mathrm{O}_{2}$ suplementario aporta indudables beneficios, aunque para ello es necesario una adecuada indicación y control de estos enfermos.

De nuestros resultados, podemos concluir que la pulsioximetría es una técnica altamente fiable, en base a su fuerte correlación con la gasometría arterial, siendo además útil para el control de la OCD, dado que es una técnica no invasiva y sobre todo se encuentra disponible en cualquier escalón asistencial.

\section{Bibliografía}

1. Sobradillo Pérez V, Miratvilles M, Gabriel R, et al. Geographic variations in prevalence and underdiagnosis of COPD: results of the IBERPOC multicenter epidemiological study. Chest 2000; 118: 981-89.

2. Comité de expertos de SEPAR. Estudio macroeconómico. Impacto social y económico de la EPOC en España. Madrid: Gabinete de Estudios Sociológicos Bernard Krief, 1995.

3. Nocturnal Oxygen Therapy Trial Group. Continuous or nocturnal oxygen therapy in hypoxemic chronic obstructive lung disease. Ann Intern Med 1980; 93: 391-98

4. Report of the Medical Research Council Working Party. Long-term domiciliary oxygen therapy in chronic hypoxic cor pulmonale complicating chronic bronchitis and emphysema. Lancet 1981; 1: 681-85.

5. Sánchez Agudo L, Cornudella R, Estopa Miró R, Molinos Martín L, Servera Pieras E. Normativa para la indicación y empleo de la oxigenoterapia continuada domiciliaria (OCD). Arch Bronconeumol 1998; 34: 87-94.

6. NHLBI/WHO Workshop. Global strategy for the diagnosis, management, and prevention of Chronic Obstructive Pulmonary Disease. National Institutes of Health. Publ. No 2701. 2001.

7. Escarrabill J, Estopá R, Huguet M, Riera J, Manresa F. Oxigenoterapia continua domiciliaria. Estudio de 344 pacientes. Arch Bronconeumol 1987; 23: 164-68.

8. León Jiménez A, Fernández Berni JJ, Gómez Gutiérrez JM, Gómez Nebreda MJ, González Moya E, Rosano Romero A. Oxigenoterapia domiciliaria: estudio de su utilización en la ciudad de Cádiz. Neumosur 1995; 7: 83-7

9. García Besada JA, Coll Artés R, Cuberta Nicolás, Padrosa Macías JM, Such Acín JJ. Oxigenoterapia crónica domiciliaria: mal uso y abuso en nuestro medio. Med Clin (Barc) 1986; 86: 527-30.

10. Jurado Gámez B, Muñoz Triano E, Davina Romero S, del Pino Gómez R, Ortiz Jiménez A, Sans Sánchez M. Oxigenoterapia domiciliaria. Estudio de utilización en una área sanitaria. Validación de la pulsioximetría. An Med Interna (Madrid) 1998; 15: 358-62.

11. Rodríguez-Roisin R, Agustí García-Navarro A, Burgos Rincón F, Casan Clará P, Perpiñá Tordera M, Sánchez Agudo L, Sobradillo Peña
V. Gasometría arterial. En Recomendaciones SEPAR. Doyma, S.A. Barcelona. 1998; 55-77.

12. Sanchis Aldás J, Casan Clará P, Castillo Gómez J, González Mangado N, Palenciano Ballesteros L, Roca Torrent J. Espirometría forzada. En Recomendaciones SEPAR. Doyma, S.A. Barcelona. 1998; 1-18.

13. Barker AF, Burgher LW, Plummer AL. Oxigen Conserving Methods for adults. Chest 1994; 105: 248-52.

14. Petty TL, O'Donohue WJ. Further recommendations for prescribing, reimbursement, technology development, and research in Long-Term Oxygen Therapy. Am J Respir Crit Care 1994; 150: 875-7.

15. Timm RM, Khaja FU, Williams GW, and the NOTT group. Haemodynamic response to oxygen therapy in chronic obstructive pulmonary disease. Ann Intern Med 1985; 102: 29-36.

16. Weitzenblum E, Sautegeau A, Ehrhart M, Mammosser M, Pelletier A Long-term oxygen therapy can reverse the progression of pulmonary hypertension in patients with chronic obstructive pulmonary disease. Am Rev Respir Dis 1985; 1317: 493-98.

17. Morrison D, Skwarski K, MacNee W. Review of the prescription of domiciliary long term oxygen therapy in Scotland. Thorax 1995; 50: 1103-5

18. Okubadejo AA, Paul EA, Wedzicha JA. Domiciliary oxygen cylinders: indications, prescription and usage. Resp Med 1994; 88: 777-85.

19. D. del Castillo, J. Sánchez, A. León, A. Arnedillo, JM. Gómez, E. González, JJ. Fernández. Eficacia de una consulta monográfica de oxigenoterapia. Neumosur 2000; 12: 73-4.

20. Muñoz Cabrera L, Jurado Gámez B, Alcázar Lanagrán B, León Jiménez A, Márquez Pérez FL, Feu Collado N, López-Campos Bodineau M. La Enfermedad Pulmonar Obstructiva Crónica en Atención Primaria. Estudio descriptivo en las comunidades de Extremadura y Andalucía. Neumosur 2001; 13: 183-191.

21. Siafakas NM, Vermeire P, Pride NB, Paoletti P, Gibson J, Howard P, et al. ERS consensus statement. Optimal assessement and management of chronic obstructive pulmonary disease (COPD). A consensus statement of the European Respiratory disease (ERS). Eur Respir J 1995; 8: 1398-420. 
22. Bujalance Zafra J, Velasco Garrido JL, Gálvez Recuero E, Martín Sánchez F, Vicente Gil P, Rueda Rios C, Rosales Jaldo M. Uso del tratamiento inhalador en nuestro medio, actual y tras aprendizaje. Neumosur 2000; 12: 73 .

23. Bosley CM, Corden ZM, Rees PJ, Cochrane GM. Phychological factors associated with use of home nebulized therapy for COPD. Eur Respir J 1996; 9: 2346-50.

24. Shim C, Williams MH. The adequancy of inhalation of aerosol from Canister Nebulizers. Am J Med 1980; 69: 891-94.

25. Fauroux B, Howard P, Muir JF, for the European Working Group on Home Treatment for Chronic Respiratory Insufficiency. Home treatment for chronic respiratory insufficiency: the situation in Europe in 1992. Eur Respir J 1994; 7: 1721-26.

26. Cottrell JJ, Openbrier D, Lave JR, Paul C, Garland JL. Home Oxygen Therapy. A comparison of 2-vs-6-month patient reevaluation. Chest 1995; 107: 358-61.

27. Escarrabill J. Oxigenoterapia domiciliaria: ¿a quién, como, cuando, donde y quien la controla?. Arch Bronconeumol 1996; 32: 1-3.

28. American Thoracic Society. Standard for the diagnosis and care of patients with chronic obstructive pulmonary disease. Am J Respir Crit Care Med 1995; 152: S77-S120.

29. Tarpy SP, Celli BR. Long-term oxigen therapy. N Engl J Med 1995; 333: 710-14.

30. Monsó E, Izquierdo J, Piqué JL, Coll R, Miró I, Morera J. Oxigenoterapia crónica y otros utillajes neumológicos de uso domiciliario en el Barcelonés Nord i Maresme. Estudio descriptivo. Arch Bronconeumol 1994; 30: 245-47.

31. Santos Luna F, Jodral Castillo T, Acasuso Díaz G, Pérula de Torres L Martínez de la Iglesia J. ¿Se emplea adecuadamente la oxigenoterapia crónica domiciliaria (OCD) en atención primaria?. Neumosur 1991; 3 : 19-22.

32. A. Granados, J. Escarrabill, JM Borrás, V. Sánchez, A.J. Jovell. Utilización apropiada y efectividad: la oxigenoterapia crónica domiciliaria en Cataluña. Med Clin (Barc)1996; 106: 251-53

33. Morrison D, Skwarshi KM, MacNee W. The adequacy of oxigenation in patients with hypoxic chonic obstructive pulmonary disease treated with long-term domiciliary oxigen. Respir Med 1997; 91: 287-91.

34. Munilla E, Carrizo S, Hernández A, Vela J, Marín JM. Oxigenoterapia continua domiciliaria en Zaragoza: estudio transversal a domicilio. Arch Bronconeumol 1996; 32: 59-63.

35. Roberts CM, Bugler JR, Melchor R, Hetzel MR, Spiro SG. Value of pulse oximetry in screening for long term oxygen therapy requirement. Eur Respir J 1993; 6: 559-62.

36. Roberts CM, Franklin J, O'Neill A, Roberts RP, Ide J, Hanley ML, et al. Screening patientts in general practice with COPD for long-term domiciliary oxygen requirement using pulse oximetry. Respir Med 1998; 92 : 1265-8.

37. Kelly AM, McAlpine R, Kyle E, How accurate are pulse oximeters in patients with acute exacerbations of chronic obstructive airways disease?. Respir Med 2001; 95: 336-40.

38. Carone M, Patessio A, Appendini L, Purro A, Czernicka E, Zanaboni S, et al. Comparison of invasive and noninvasive saaturation monitoring in prescribing oxygen during exercise in COPD patients. Eur Respir J 1997; 10: 446-51.

39. Murray RP, Anthonisen NR, Connett JE, Wise RA, Lindgren PG, Greene PG, Nides MA. Effects of multiple attempts to quit smoking and relapses to smoking on pulmonary function. Lung Health Study Research Group. J Clin Epidemiol 1998; 51: 1317-26. 